\title{
Las algias como factor predisponente de lesión en estudiantes de baile flamenco
}

\author{
Algias as a predisposing factor of injury in flamenco dance students
Irene Baena-Chicón ${ }^{1,2,4}$, Sebastián Gómez-Lozano ${ }^{1}$, Lucía Abenza-Cano ${ }^{3}$, Olaia Abadía-García de Vicuña ${ }^{4}$, M. Rosario Fernández-Falero ${ }^{5}$, Alfonso Vargas-Macías ${ }^{4,6}$

1 Grupo de Investigación en Artes Escénicas GIAE. Facultad de Deporte. Universidad Católica San Antonio de Murcia. España.

2 Conservatorio Profesional de Danza Pepa Flores de Málaga. España.

3. Grupo de Investigación ARETÉ. Facultad de Deporte. Universidad Católica San Antonio de Murcia. España.

4 Centro de Investigación Flamenco Telethusa. España.

5 Departamento de Información y Comunicación. Universidad de Extremadura. España.

6 Grupo de Investigación Hermes CTS601. Universidad de Sevilla. España.

CORRESPONDENCIA:

Sebastian Gómez-Lozano

sglozano@ucam.edu

Recepción: enero 2020 • Aceptación: febrero 2020
CÓMO CITAR EL ARTÍCULO:

Baena-Chicón, I., Gómez-Lozano, S., Abenza-Cano, L., Abadía, García-De Vicuña, O., Fernández-Falero, M.R., \& Vargas-Macías, A. (2020). Las algias como factor predisponente de lesión en estudiantes de baile flamenco. Cultura, Ciencia y Deporte, 15(44), 245-253.

\section{Resumen}

El baile flamenco es una profesión con elevado riesgo de lesiones debido, entre otros factores, a las exigentes demandas de esfuerzo y a las vibraciones derivadas de los impactos del zapateado. El objetivo de este estudio fue determinar la prevalencia de antecedentes de algias y lesiones en estudiantes de baile flamenco en las enseñanzas artísticas profesionales para conocer el grado de relación del dolor como factor predisponente de lesión. La muestra analizada consta de 75 alumnas de Conservatorios Profesionales de Danza de Andalucía (17.4 \pm 4.4 años). Se realizó una entrevista directa basada en preguntas dicotómicas sobre la aparición de antecedentes de algias y lesiones en 7 conjuntos articulares: pies, tobillos, rodillas, coxo-femorales, columna lumbar, columna cervical y hombros. A diferencia de otras danzas percutivas, las participantes mostraron una mayor incidencia de alteraciones funcionales y de lesiones tanto en rodillas como en columna lumbar y cervical. Posiblemente, esta diferencia sea debida a que la técnica del zapateado flamenco requiere de una semiflexión de rodillas y no integra saltos o basculaciones verticales que permitan disipar las vibraciones derivadas de los impactos.

Palabras clave: dolor, zapateado, riesgo, conservatorio.

\section{Abstract}

Flamenco dancing is a high-risk profession of elevated risk of injury due, among other factors, to high stress demands and vibrations derived from the damage caused by footwork step. The objective of this study was to determine the prevalence of the antecedents of pain and the consequences of injuries in flamenco dance student from Professional Dance Conservatory. The sample analyzed consists of 75 students of Professional Dance Conservatories of Andalusia (17.4 \pm 4.4 years of age). A direct interview was applied based on dichotomous questions about antecedents of pain and injuries in 7 joint regions: feet, ankles, knees, hips, lumbar spine, cervical spine and shoulders. Unlike other percussive dances, participants showed a higher incidence of functional disorders and injuries in knees, lumbar and cervical spine. Perhaps, this difference is because flamenco footwork technique requires a semi-flexion of knees, which does not integrate vertical jumps or tilts, these would allow dissipating the vibrations derived from the impacts.

Key words: pain, footwork step, risk, conservatory. 


\section{Introducción}

El baile flamenco es una danza que tiene su origen en las regiones del sur de España. Se caracteriza por una fuerte impronta emocional y por su técnica de zapateado que le hace genuina y totalmente diferente al resto de danzas percutivas (Baena-Chicón, 2016; Pedersen \& Wilmerding, 1998). Los altos niveles de esfuerzo que demanda la interpretación del baile flamenco son similares a las observadas en actividades físico-deportivas de élite (Pedersen, Wilmerding, Kuhn, \& Enciñias-Sandoval, 2001; Vargas, 2009). En este sentido, estos practicantes, junto a los de danza clásica, son considerados como deportistas de élite (Koutedakis \& Jamurtas, 2004; Vargas, González, Mora, \& Lozano, 2008). Se ha comprobado que, durante una actuación profesional, los bailaores realizan un promedio de cuatro zapateados por segundo, pudiendo alcanzar frecuencias de hasta doce zapateados por segundo (Vargas, 2009). Este hecho físico-artístico requiere de altas demandas de esfuerzo con sus consiguientes riesgos de lesión, focalizado principalmente en pies, rodillas y espalda, principalmente a nivel lumbar y cervical (Vargas-Macías, Castillo, \& Fernández, 2012). Los suelos inadecuados que no facilitan la absorción del impacto del zapateado, el tipo de calzado (Figura 1), la altura del tacón, la dificultad de la coreografía y un calentamiento insuficiente son otros de los factores que pueden provocar riesgo de lesión en esta actividad (Castillo-López, Palomo, Munuera-Martínez, Ramos-Ortega, \& Domínguez-Maldonado, 2015; Koutedakis \& Jamurtas, 2004; Vargas, 2009). El Instituto Nacional de Seguridad e Higiene en el Trabajo ha declarado que la interpretación artística del baile flamenco es una profesión de alto riesgo de lesiones de miembro inferior (Nogareda, Salas, \& Parra, 2007).

La práctica del zapateado es un gesto técnico no natural, para el que las articulaciones del miembro inferior y columna no están diseñadas (Pedersen et al, 1998), aunque una técnica adecuada y una buena preparación física puede atenuar el efecto lesivo del zapateado (Castillo-López et al., 2015; Quer \& Pérez, 2004; Vargas, 2009). El impacto de esta actividad repetitiva incrementa el riesgo de lesión por sobreuso y estrés repetitivo (Shybut, Rose, \& Strongwater, 2008). Este hecho afecta de forma directa a aquellas articulaciones y zonas que chocan directamente contra el suelo y, además, a las diferentes estructuras corporales que en sentido ascendente absorben las vibraciones generadas por el impacto (Castillo-López et al., 2015; Quer et al., 2004; Vargas, 2009). Dicha vibración en ocasiones puede ser el factor desencadenante de algias vertebra-

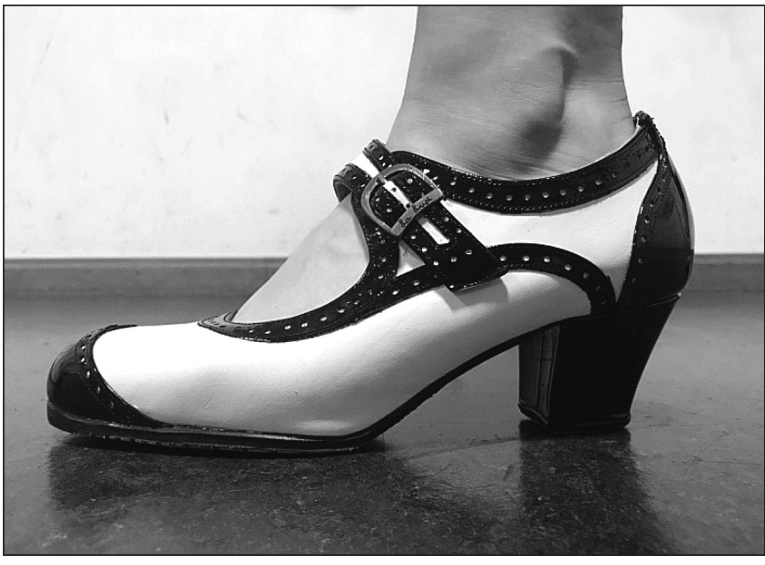

Figura 1. Calzado de tacón tradicional de baile flamenco

les, tanto lumbares como dorsales o cérvico-craneales. Las algias o dolor localizado en una región son signo e indicador de aviso de lesión (Gómez, Santonja, Vargas, \& Canteras, 2010). Es notorio que los practicantes de baile flamenco son muy susceptibles de padecer dolor (Castilla-Cubero \& Giménez-Sarmiento, 2011). Este carácter de expresión de sufrimiento que acompaña al arte flamenco debe alertar a los profesionales de la salud para generar mecanismos en la prevención de lesiones.

Hasta donde nuestro conocimiento alcanza, no existen manuales adecuados y específicos para la formación del baile flamenco en el ámbito del acondicionamiento técnico y prevención de lesiones. Armstrong y Davies (1984) afirman que los deportistas logran mejores resultados si en la infancia o adolescencia han realizado programas de entrenamiento sistematizados y bien organizados. En el arte flamenco, incluido el baile, el conocimiento de esta práctica se ha producido a través de la transmisión oral de generación a generación, de maestros a alumnos, en academias y entre familiares (Baena-Chicón, 2016). Desde el Siglo XIX, este tipo de danza alberga una larga experiencia formativa en salones y otros espacios privados, la cual se mantiene actualmente por la expansión de academias de flamenco por todo el mundo (Navarro \& Pablo, 2005). En España, además de en estos lugares, se puede estudiar baile flamenco en algunas escuelas municipales y en determinados conservatorios profesionales de danza.

Gracias a que se establecen los aspectos curriculares en el Real Decreto 85/2007, de 26 de enero, se introduce oficialmente la especialidad de baile flamenco como enseñanza profesional de danza en España. Actualmente se puede estudiar en conservatorios de Andalucía, Madrid y Murcia. En este ámbito formativo, la finalidad de este nivel educativo es la de proporcionar al alumnado una formación artística de cali- 
dad, así como garantizar la cualificación de los futuros profesionales de la danza (Heras, 2010). Cuentan con asignaturas, entre otras, de Técnicas básicas de danza (danza clásica y contemporánea) y danza española para complementar la formación dancística y proporcionarle una mejor condición física.

Encarnación, Meyers, Ryan y Pease (2000) afirman que, en el ballet, existe una tendencia en no prestar atención a la lesión y seguir bailando. De hecho, estos artistas son enseñados desde una temprana edad a que el ballet es equivalente a dolor (Paparizos, Tripp, Sullivan, \& Rubenstein, 2004; Rivera, Alexander, Nehrenz, \& Fields, 2012). En nuestro estudio, el objetivo es determinar la prevalencia de antecedentes algias y lesiones en estudiantes de baile flamenco en las enseñanzas artísticas profesionales para conocer el grado de relación del dolor como factor predisponente de lesión.

\section{Método}

\section{Participantes}

En el estudio han participado 75 alumnas de las enseñanzas profesionales de danza, en la especialidad de baile flamenco, de Conservatorios Profesionales de Danza de Andalucía. La edad media de las participantes es de $17.4 \pm 4.4$ años, con una experiencia en el baile flamenco de $11.5 \pm 4.5$ años que dedicaban una media de 8:22 \pm 3:33 horas de baile flamenco a la semana. Algunas sugerencias metodológicas de diferentes autores (Abenza, Olmedilla, Ortega \& Esparza, 2009; Petrie \& Falkstein, 1998) impulsaron la elección de una muestra homogénea (mismo sexo, misma especialidad y mismo nivel educativo y horas de práctica) para profundizar en la investigación sobre dolor y lesiones derivadas del ejercicio físico sistematizado.

Instrumentos y procedimiento de recogida de datos

Se realizó una entrevista directa basada en preguntas dicotómicas sobre la aparición de antecedentes de algias y lesiones en 7 conjuntos articulares: pies, tobillos, rodillas, coxo-femorales, columna lumbar, columna cervical y hombros.

En cuanto al diseño, desde el punto de vista de la metodología de encuestas, es un diseño transversal. Todas las bailaoras encuestadas dieron su consentimiento para responder el cuestionario y, en el caso de las menores de edad, los padres firmaron el consentimiento informado.

\section{Análisis estadístico}

Se realizó un análisis descriptivo con la obtención de los parámetros característicos, media y desviación típica, para las variables cuantitativas mediante el programa estadístico SPSS 16.0. Se aplicó un análisis del contraste de igualdad de dos o de varias proporciones a través de un análisis de la tabla de contingencia con la prueba de Chi-cuadrado de Pearson para comparar el grado de aparición de lesiones articulares y para determinar el grado de asociación entre el dolor con el de lesión de cada una de las articulaciones. Para medir el factor de asociación de riesgo entre la aparición de dolor en una articulación con la probabilidad de sufrir lesión en esa misma zona, se aplicó un análisis de tablas de contingencia y el cálculo de odds ratio (OR).

\section{Resultados}

El 74.7\% de los sujetos reconocen que sienten dolor durante la práctica de baile flamenco y el $66.7 \%$ de la muestra afirma que han estado lesionados alguna vez.

Se determina una asociación significativa en cualquier articulación de la cadena postural excepto en la región del pie ( $\chi 2$ = 2.142; $p=0.405)$. Tanto en la articulación del tobillo $(\chi 2=7.609 ; p=0.003)$, rodilla $(\chi 2=15,074$; $\mathrm{p}<0,00005)$, coxo-femoral $\left(\chi^{2}=24.324 ; \mathrm{p}<0.00005\right)$, región lumbar ( $\chi 2=4.553 ; \mathrm{p}=0.01)$, región cervical ( $\chi 2$ $=8.858 ; \mathrm{p}=0.001)$ como en el conjunto articular de los hombros ( $\chi 2=7.246 ; \mathrm{p}=0.003$ ) se puede apreciar diferentes grados de relación significativa.

En consecuencia, se establecen el número de casos de prevalencia de algias y lesiones para cada una de las articulaciones (Tabla 1).

Tabla 1. Resultados sobre el grado de asociación intra-articular entre algias y lesiones.

\begin{tabular}{lcc}
\hline & $\begin{array}{c}\text { Algias } \\
\mathbf{n}=75\end{array}$ & $\begin{array}{c}\text { Lesiones } \\
\mathbf{n}=75\end{array}$ \\
\hline Pie & $28(37.3 \%)$ & $15(20 \%)$ \\
Tobillo * & $8(10.7 \%)$ & $24(32 \%)$ \\
Rodillas* & $28(37.3 \%)$ & $25(33.3 \%)$ \\
Coxo-femoral ** & $3(4 \%)$ & $1(1.3 \%)$ \\
Lumbar * & $24(32 \%)$ & $12(16 \%)$ \\
Cervicales * & $19(25.3 \%)$ & $17(22.7 \%)$ \\
Hombro * & $11(14.7 \%)$ & $9(12 \%)$ \\
\hline
\end{tabular}

Valores con grado de asociación significativa $(*)$ y muy significativa $(* *)$ para $\times 2$.

A nivel inter-articular (Tabla 1) se vislumbra una relación significativa entre las variables antecedentes de dolor de pie con la lesión de tobillos y lesión de lumbar $(\chi 2=2,693 ; p=0,05)$. También se establece una 
relación significativa entre antecedentes de dolor de rodillas con la lesión cervical $(\chi 2=4,339 ; \mathrm{p}=0,02)$ y antecedentes de dolor cervical tanto con la lesión de tobillos ( $\chi 2=2,762 ; \mathrm{p}=0,05)$ como con la lesión coxofemoral $\left(\chi^{2}=2,987 ; \mathrm{p}=0,04\right)$ y lesión lumbar $\left(\chi^{2}=\right.$ $8,225 ; \mathrm{p}=0,002)$.

En la Tabla 2 se muestran las zonas articulares que han obtenido relaciones significativas como factores del riesgo de antecedentes de algias previas y el aumento proporcional de la probabilidad de sufrir lesiones en base a la odd ratios (OR).

Tabla 2. Odd Ratios hallados para la relación entre el hallazgo de antecedentes de dolor y probabilidad de riesgo de lesión.

\begin{tabular}{llcc}
\hline Dolor zona articular & Lesión zona articular & OR & $\mathbf{9 5 \%} \mathbf{C I}$ \\
\hline Pie & Tobillo & 2.2 & $0.8-5.9$ \\
Pie & Lumbar & 2.8 & $0.8-9.9$ \\
Tobillo & Tobillo & 8.2 & $1.5-44.2$ \\
Rodilla & Rodilla & 7.5 & $2.6-22.1$ \\
Rodilla & Cervical & 3.2 & $1.1-9.7$ \\
Coxo-femoral & Coxo-femoral & 1.5 & $0.7-3.3$ \\
Lumbar & Lumbar & 3.8 & $1.1-13.6$ \\
Cervical & Tobillo & 2.5 & $0.8-7.2$ \\
Cervical & Coxo-femoral & 1.1 & $0.9-1.2$ \\
Cervical & Lumbar & 5.9 & $1.6-22.1$ \\
Cervical & Cervical & 5.4 & $1.7-17.4$ \\
Hombro & Hombro & 6.7 & $1.5-31.2$ \\
\hline
\end{tabular}

\section{Discusión}

La finalidad de esta investigación es analizar, de manera retrospectiva, los antecedentes de algias y lesiones en estudiantes de conservatorios de baile flamenco de Andalucía, donde las enseñanzas artísticas de esta disciplina forman parte de la idiosincrasia cultural. Dicho análisis puede revelarnos información útil para poder establecer estrategias en el futuro con las practicantes de baile flamenco en la gestión tanto de estas propias alteraciones funcionales como del propio entrenamiento. La aparición de estas alteraciones nos ayuda a entender mejor tanto la técnica de zapateado (Pedersen et al., 1998) como del resto de elementos que convergen en la propia estética del baile flamenco. Una adecuada indicación técnica, táctil o verbal, es de gran utilidad para mejorar posibles sobrecargas, descompensaciones y retracciones tendinoso-musculares, previas a patologías o lesiones de cierta gravedad (Hoogenboom \& Voight, 2017).

El porcentaje de antecedentes de algias es relativamente bajo en la articulación coxo-femoral (4\%). En Castilla-Cubero et al. (2011) las coxalgias también obtienen valores mínimos de $17,7 \%$ de su muestra, seguidos sólo por el dolor de manos $13.5 \%$ y codos
$4.2 \%$. Esta coincidencia ocurre porque en el flamenco no se trabaja tanto la flexibilidad de las caderas como en bailarines de danza clásica (Baena-Chicón, VargasMacías, \& Gómez-Lozano, 2015). Posiblemente esta falta de trabajo de flexibilidad en el flamenco evite una desestabilización músculo-articular de cadera que puede llevar en poblaciones como las del ballet a la displasia acetabular (Weber, Bedi, Tibor, Zaltz, \& Larson, 2015) y otras patologías de cadera (Turner, O’Sullivan, \& Edelstein, 2012), aunque a largo plazo posiblemente pueda incidir en otro tipo de deformación o alteraciones por un exceso de tensión alrededor de esta articulación en la cadera del intérprete de baile en flamenco ya en edad adulta.

El conjunto articular pies y rodillas obtiene el mayor porcentaje de antecedentes de algias de entre todas las zonas estudiadas, en concreto un $37.3 \%$. Estos resultados son similares a los registrados por Vargas (2009) entre bailaoras profesionales de baile flamenco donde un $27.3 \%$ de las participantes manifestaban dolor tanto en pies como en rodillas. Por otro lado, Castilla- $\mathrm{Cu}-$ bero et al. (2011) elevan hasta un 50\% los casos dolor en rodillas y hasta un $61.45 \%$ en pies, mientras que Castillo-López et al. (2014) encuentran el 80.7\% de las bailaoras profesionales estudiadas manifestaban dolor metatarsal mientras bailaban y el $34.78 \%$ a nivel patelofemoral. Esta evidencia sobre los pies y rodillas como zonas más dolorosas está contrastada en otros estudios (Bejjani, 1987; Calvo, Alonso, Pasadolos, \& Gómez-Pellico, 1998; Quer et al., 2004; Shybut et al., 2008) donde la acción de zapatear y el uso del zapato de tacón son las primeras causas.

Respecto a la presencia de lesión, se observa que las articulaciones coxo-femorales presentan la menor incidencia de todas las zonas estudiadas, en concreto un $1.3 \%$, valor similar a los resultados obtenidos por Echegoyen, Acuña, y Rodríguez (2010) donde un $1.08 \%$ se localiza en osteítis púbica. Estos resultados son inferiores a los registrados en el ballet, para las articulaciones coxo-femorales que oscilan entre un 9.9\% (Reid, 1988) y un $13.4 \%$ (Sobrino \& Guillén, 2017). Esta diferencia puede estar justificado por la rotación externa de caderas, turnout o en dehors, elemento técnico esencial en el ballet y que está presente en el $100 \%$ de sus coreografías, mientras que en el baile flamenco su relevancia es mucho menor y es usado solamente en un 25.53\% de los espectáculos (Baena-Chicón et al., 2015). También son bastante inferiores a los datos resgistrados en otras danzas percutivas. En el claqué, la cadera muestra una incidencia de lesión del 9\% de los casos (Mayers, Judelson, \& Bronner, 2003) y en la danza irlandesa oscila entre el $12 \%$ y el $16 \%$ de los casos (Eustergenling \& Emery, 2015; McGuin- 
ness \& Doody, 2006). Las diferencias pueden estar justificadas por cuestiones técnicas y estéticas de estas danzas percutivas. Los impactos sonoros en el claqué y danza irlandesa se realizan coordinados con pequeños brincos y saltos propios de estas danzas, donde la flexo-extensión de caderas juega un papel fundamental y por tanto su rol en la ejecución y abosorción de impactos también. En cambio, en el baile flamenco no se realizan pequeños saltos sino que el impacto sonoro del zapato se produce principalmente por una flexoextensión de rodillas. Por ello el papel percutivo que juega la cadera es menor que en el claqué y la danza irlandesa.

Las rodillas, con un $33.3 \%$, son las zonas que manifiestan mayores casos de lesiones en nuestra muestra flamenca. Estos datos coinciden con los datos registrados por Pedersen et al. (1998) entre profesionales del baile flamenco, un $30 \%$, pero muy superiores al $15 \%$ registrado entre bailaores/as estudiantes de ese mismo estudio. Sobrino et al. (2017) reducen la incidencia registrada de lesión en rodillas al $22.4 \%$ de su muestra profesional. Por el contrario, estos datos son superiores a los obtenidos en estudiantes de otras danzas percutivas donde el porcentaje de lesiones oscila entre un $10 \%$ para la danza irlandesa (Eustergenling et al., 2015; McGuinness et al., 2006) y un 14\% para el claqué (Mayers et al., 2003). Al realizarse el zapateado flamenco con una flexo-extenisón de rodillas, esta articulación no solo desempeña un rol percutor, sino que técnicamente solo es posible si mantiene una semiflexión permanente. Esta larga exposición aumenta la fuerza comprensiva en la rótula por lo que sobrecarga excesivamente la zona femoropatelar (Calvo et al., 1998; Castillo-López, Munuera-Martínez, GómezBenítez, Pérez-García, Salti-Pozo, \& Palomo, 2017).

Los tobillos, con un $32 \%$, es la segunda zona articular donde se han recogido más casos de lesión. Similares resultados han sido registrados en estudiantes de otras danzas percutivas, en concreto un $26 \%$ para claqué (Mayers et al., 2003) y de $30 \%$ y $32 \%$ para danza irlandesa (Eustergenling et al., 2015; McGuinness et al., 2006). Resulta significativo que en profesionales del baile flamenco los porcentajes hallados sean algo menores, entre un 23\% y 25\% (Pedersen et al., 1998; Sobrino et al., 2017).

Los pies son otra zona de alta incidencia de lesión, en concreto se ha registrado un $20 \%$ de los casos. Resultados similares han sido hallados entre profesionales de baile flamenco (Pedersen et al., 1998) y estudiantes de danza irlandesa (Eustergenling et al., 2015; McGuinness et al., 2006). Como consecuencia de golpear rítmicamente y con musicalidad el suelo, el pie no solo adopta un rol percutor, sino que asume todas las cargas de ese golpe. Además, el calzado flamenco no está diseñado para absorber impactos ni las superficies de bailes siempre son las más idóneas (CastilloLópez, Munuera-Martínez, Algaba-Guisado, ReinaBueno, Salti-Pozo, \& Vargas-Macías, 2016). Todo ello conlleva que más del $80 \%$ de las profesionales del baile flamenco presenten deformaciones de carácter podológico (Castillo et al., 2014).

La columna vertebral presenta también altas incidencias de lesiones, tanto a nivel lumbar como cervical. Los resultados del presente estudio son similares a los registrados en profesionales del baile flamenco (Pedersen et al., 1998). Los mecanismos biomecánicos de zapateados tienen una repercusión sobre la cadena cinética musculo-articular muy similar. El impacto del zapato se transmite por ondas vibracionales desde las articulaciones del tren inferior hasta la columna vertebral, lo cual puede desencadenar algias vertebrales (Gómez, et al., 2010), sobrecarga en la musculatura de la espalda (Echegoyen et al., 2010) y lesiones en el tren inferior (Pedersen et al., 1998). Resulta significativo que las lesiones registradas en la espalda sean bastante menores en estudiantes de otras danzas percutivas, en concreto un 5\% para la danza irlandesa (McGuinness et al., 2006) y un 14\% para el claqué (Mayers et al., 2003). Estas danzas integran saltos en la percusión del pie, así se disipan de forma más eficiente las vibraciones del impacto. En cambio, en el flamenco, al no participar el tren superior con evidentes movimientos ascendentes y descendentes, se obliga a las estructuras de la espalda a absorber las vibraciones que no han sido disipadas en esta basculación. Cabe señalar que durante las fases de zapateado los desplazamientos verticales del centro de gravedad son muy reducidos, de solo $1.8 \mathrm{~cm}$ (Forczek, Baena-Chicón, \& Vargas-Macías, 2016).

Los hombros son, tras las caderas, una de las zonas con menor incidencia de lesión. Los datos son similares a los registrados por profesionales del baile flamenco (Sobrino et al., 2017) y la danza irlandesa (McGinness et al. 2006). Es posible que las lesiones en esta zona de los bailaores de flamenco no sean consecuencia de los zapateados, sino más bien de los movimientos del tren superior durante las fases de braceo del baile.

Son escasos los estudios acerca de la experiencia de los procesos sobre gestión del dolor en la danza (Encarnacion et al., 2000; Mainwaring \& Finney, 2017; Prokop, 2000) y muy pocos en practicantes de baile flamenco (Vargas, 2009). Es importante analizar la relación entre el dolor y la aparición de una lesión, ya que el dolor es un signo de alarma que avisa de la calidad de la función motriz y su desestabilización (Anderson \& Hanrahan, 2008) a corto y a largo plazo. 
Actualmente, es en verdad complejo realizar un seguimiento a bailaores en relación al dolor como a la evolución de una lesión. Su umbral y su nivel de tolerancia al dolor es más alto que los que no practican danza (Russell, 2013). Este hecho puede justificar que no se correlacione los informes de dolor con los hallazgos de lesión mediante radiodiagnóstico (Duthon et al., 2013; Russell, Shave, Yoshioka, Kruse, Koutedakis, \& Wyon, 2010). Anderson et al. (2008) hallaron que los bailarines de ballet tendían a distinguir mal entre el dolor habitual en la danza y el dolor asociado con lesiones.

Tanto el miedo a ser reemplazado en una actuación como la incorporación a su colectivo imaginario de la sentencia el espectáculo debe continuar (Anderson et al., 2008) conlleva a que, a pesar del dolor, los bailarines tienden a continuar bailando para mantener la condición física, la técnica y el estatus dentro del grupo. Esto implica que muchas de las algias deriven en lesión, pues sus habilidades para gestionar eficientemente el dolor no están tan desarrolladas como ocurre en otros deportes (Encarnacion et al., 2000).

En este estudio, determinamos una asociación intra-articular de manera general muy significativa entre dolor y lesión, en toda la cadena de articulaciones que conforman la postura del sujeto (Tabla 1), excepto en el pie, a pesar de las altas incidencias de algias registradas. Esto puede ser debido a que las sobrecargas del pie en el baile flamenco se traducen sobre todo en hallux abductus valgus, hiperqueratosis, onicopatías, onicodistrofias, dedos en garra o juanete de sastre (Castillo, Pérez, \& Algaba, 2010; Castillo, Palomo, Munuera, Domínguez, Algaba, \& Pérez, 2011; Castillo, Munuera, Domínguez, Salti, \& Algaba, 2013; Castillo-López et al., 2016), deformaciones podológicas que son asumidas como inherente al baile y no interpretadas como lesión.

En las articulaciones que reflejan una asociación significativa más fuerte entre dolor y lesión son las rodillas y la coxo-femoral. El uso de los zapatos de tacón alto es uno de los factores que favorecen la aparición de alteraciones en el aparato locomotor (Castillo et al., 2010; Castillo et al., 2011; Castillo et al., 2013; Castillo-López et al., 2016; Pedersen et al., 1998). Es conocido que el impacto del zapateado afecta directa e indirectamente sobre el conjunto articular de las extremidades inferiores Bejjani, Halpern, Pio, Domínguez, Voloshin, \& Frankel, 1988; Voloshin, Bejjani, Halpern, \& Frankel 1989) y que un alineamiento incorrecto de la rodilla en relación con el pie durante la semiflexión del zapateado puede originar el dolor y posterior lesión en esta articulación (Snow \& William, 1994). Pedersen et al. (1998), alude a que una ines- tabilidad articular provoca distintas desviaciones de alineamiento que afectan a la capacidad corporal para absorber los impactos.

Aunque la articulación coxo-femoral tiene una proporción significativa bastante fuerte, el porcentaje de bailaoras que sufren dolores es muy baja, lo que nos permite suponer que los mecanismos desencadenantes de lesiones en esta articulación son ocasionados por fatiga crónica a largo plazo, donde los signos previos no aparecen hasta cierta edad adulta.

Cuando analizamos la relación de afectación entre articulaciones se establece una proporción significativa entre antecedentes de dolor de pie con las lesiones de tobillo y lumbar. El pie es la primera estructura del aparato locomotor, en contacto con el suelo. Su relación con la aparición de lesiones tanto en tobillos como en la región lumbar nos indica claramente la importancia de la alineación de segmentos durante el zapateado en el baile flamenco. Es conocido que la inestabilidad del pie durante las fases de zapateado es frecuente en el baile flamenco (Vargas et al., 2012). Dicho aspecto, unido al hecho de que los impactos no sean amortiguados con brincos como en otras danzas percutivas, vincula el dolor de la estructura pie-tobillo con la región lumbar.

El uso de tacón alto en esta práctica puede llegar a ser el desencadenante de la mayoría de las alteraciones de aparato locomotor que se presentan en el baile flamenco (Castillo-López et al., 2016) Se ha demostrado que este tipo de calzado no absorbe mayoritariamente los impactos ocasionados por la percusión del zapato contra el suelo (Echegoyen, Aoyama, \& Rodríguez, 2013; Vargas, 2009; Voloshin et al., 1989). Las señales que aparecen de dolor del pie advierten de potenciales lesiones, ya que el bailaor de manera inconsciente compensa reestructurando su colocación. De esta manera, los impactos se pueden ver gestionados con ciertas desviaciones angulares. Es el caso de la pronación y supinación del pie o de la lordosis lumbar provocados por la inestabilidad que produce los zapatos de tacón. Así, cuando se impone una mayor demanda sobre los músculos gastrocnemio y sóleo, que se encuentran acortados por la altura del tacón, precisan de un alargamiento y disminución de la dependencia del tibial anterior correspondiente (Pedersen et al., 1998). Una inestabilidad ocasionada por el dolor puede afectar a toda la cadena cinetico- muscular hasta la región lumbar de manera significativa (Swain, Bradshaw, Ekegren, \& Whyte, 2019).

En este sentido ascendente articular se aprecia una asociación de afectación y lesión cervical previo dolor de rodillas. Las situaciones continuadas de flexión de rodillas, tal y como sucede durante el zapateado, ha 
sido determinada como causante de problemas vertebrales a nivel lumbar (Omino \& Hayashi, 1992) como cervical (Yoo, 2013).

El sentido cefálico del binomio dolor-lesión se invierte en sentido caudal cuando se ve afectada la región cervical. Las cervicalgias van a favorecer la lesión en tobillos, coxo-femorales y en región lumbar. Esta correlación puede derivar principalmente por la vinculación existente entre el golpe de zapateado y las vibraciones del impacto. Una de las consecuencias de zapatear con un calzado que no posee ningún sistema de amortiguación es que las estructuras del pie y tobillo sufran lesiones como consecuencia directa del golpe. Este impacto produce vibraciones que ascienden hasta la parte superior de la cabeza originando sobrecargas musculares en la zona cervical, ya que según la estética flamenca el torso se mantiene lo más estático posible y no amortigua impactos con desplazamientos verticales. Estos dolores cervicales podrían prevenirse con una preparación física específica de carácter preventivo y manipulaciones de descarga muscular de la zona (Vargas, 2009).

Se observa que la salud de todo el sistema locomotor se encuentra comprometida, por diversos factores que afectan al practicante de flamenco. Principalmente avisado por signos de dolor, ciertas estructuras articulares y musculo-tendinosas pueden llegar a lesionarse gravemente. El riesgo probable de lesión (OR) previo dolor se manifiesta principalmente en el tobillo, rodilla y región lumbar (Tabla 2).

Como expusieron Taylor y Taylor (1998) el dolor de rendimiento que sienten los deportistas es como el dolor benigno, por lo que están acostumbrados a forzar y seguir los entrenamientos hasta que con el sobreesfuerzo caen lesionados. Los bailarines tienen que dominar unas técnicas muy complejas que requieren unas altas demandas físicas muy específicas y, en consecuencia, se requiere un exigente entrenamiento físico (Anderson et al., 2008; Mainwaring, Hutchison, Bisschop, Comper, \& Richards 2010) que indica que los bailarines suelen ver el dolor como un compañero de la práctica de la danza, siendo propensos a "bailar a través del dolor", incluso cuando hacerlo puede ser perjudicial. Por ello, resulta coherente esta alta probabilidad de riesgo de lesión en todos los resultados obtenidos.

Se hallan varias zonas sensibles y susceptibles de ser alteradas en su funcionamiento. A nivel apendicular inferior: tobillos y rodillas, descartando la región coxo-femoral, ya que al no analizar a sujetos en edad adulta o mayores de 40 años no podemos estudiar las consecuencias degenerativas de una vida entera dedicada al flamenco.
A nivel axial encontramos la región cervical y lumbar como dos curvas fundamentales y muy sensibles en el mantenimiento de la técnica del practicante de flamenco. Las dos curvas juegan un papel determinante en la salud integral del sujeto.

En el ámbito académico es fundamental preservar la salud integral de los discentes, garantizar una dilatada carrera profesional y optimizar su rendimiento artístico. En este sentido, conocer los indicadores de dolor que actúan como predictores de lesión, resulta vital para implementar estrategias de prevención o adaptaciones metodológicas que no comprometan el bienestar de las bailaoras.

\section{Conclusiones}

Los valores de antecedentes de algias son superiores a los de lesión. Posiblemente sea debido, a que los estudiantes de baile flamenco generan un proceso de adaptación a la sensación de dolor, ya que está asimilado como algo propio debido a su herencia cultural. Este hecho parece determinar que las articulaciones tanto del tren inferior como de la columna vertebral son más susceptibles de sufrir lesiones o alteraciones de mayor gravedad.

Los resultados sobre la prevalencia en lesiones son similares a otros estudios sobre baile flamenco, ya que parece ser explícito que los elementos técnicos que marcan el estilo son siempre factores de riesgo. Una de las características diferenciadoras del baile flamenco dentro de las danzas percutivas es la ausencia de brincos acompasados al zapateado, hecho que parece tener relación directa con una mayor incidencia de alteraciones funcionales y lesiones tanto en rodillas como en la columna lumbar y cervical.

Es evidente la alta exigencia del baile flamenco durante el zapateado, más aún en un entorno reglado donde los estudiantes tienen una carga lectiva considerable en los Conservatorios Profesionales de Danza.

Es recomendable la creación de una normativa en este ámbito educativo que regule tanto el calzado como los suelos de estos centros artísticos de enseñanza.

\section{Agradecimientos}

Los autores desean mostrar su agradecimiento a los Conservatorios Profesionales de Danza de Andalucía por su colaboración para implementar los cuestionarios entre sus estudiantes de baile flamenco que nos han permitido llevar a cabo el presente estudio. 


\section{BIBLIOGRAFÍA}

Abenza, L., Olmedilla, A., Ortega, E., \& Esparza, F. (2009). Lesiones y factores psicológicos en futbolistas juveniles. Archivos de Medicina del Deporte, 26(132), 280-288.

Anderson, R., y Hanrahan, S. J. (2008). Dancing in pain: pain appraisal and coping in dancers. Journal of Dance Medicine y Science, 12(1), 9-16.

Armstrong, N., y Davies, B. (1984). The metabolic and physiological res ponses of children to exercise and training. Phys Educ Rev, 2, 90-105.

Baena-Chicón, I. (2016). Tratamiento coreográfico en el baile Flamenco. Revista del Centro de Investigación Flamenco Telethusa, 9(11), 11-14.

Baena-Chicón, I., Vargas-Macías, A., \& Gómez-Lozano, S. (2015). Análisis diacrónico y descriptivo del en dehors en el baile flamenco. Revista del Centro de Investigación Flamenco Telethusa, 8(9), 19-28.

Bejjani, F. J. (1987). Occupational biomechanics of athletes and dancers: a comparative approach. Clinics in podiatric medicine and surgery, 4(3), 671-711.

Bejjani, F. J., Halpern, N., Pio, A., Domínguez, R., Voloshin, A., \& Frankel, V. H. (1988). Musculoskeletal demands on flamenco dancers: a clinical and biomechanical study. Foot y ankle international / American Orthopaedic Foot and Ankle Society [and] Swiss Foot and Ankle Society, 8(5), 254-263. https://doi.org/10.1177/107110078800800505

Calvo, J., Alonso, A., Pasadolos, A., \& Gómez-Pellico, L. (1998). Flamenco Dancing. Biomechanical Analysis and Injuries Prevention. En En: Macara, A., editor. Continents in movement. Proceeding of the Internacional Conference. New trends in dance teaching. (pp. 279-285). Oeiras: M. H. Ediçoes. Recuperado de http://journal.unair.ac.id/downloadfullpapers- $\ln 522 c c 87 c 61$ full.pdf

Castillo, J. M., Munuera, P. V., Domínguez, G., Salti, N., \& Algaba, C. (2013). Prevalencia del juanete de sastre y quinto dedo adducto varo en el baile flamenco profesional. Revista del Centro de Investigación Flamenco Telethusa, 6(7): 13-18.

Castillo, J.M., Palomo, I.C., Munuera, P.V., Domínguez, G., Algaba, C., \& Pérez, J. (2011). El Hallux Abductus Valgus en la bailaora de flamenco. Revista del Centro de Investigación Flamenco Telethusa, 4(4), 19-24.

Castillo, J.M., Pérez, J., \&Algaba, C. (2010). Estudio preliminar. Patologías digitales más frecuentes en el pie de la bailaora de flamenco. Revista del Centro de Investigación Flamenco Telethusa, 3 (3): 32-35

Castilla-Cubero, J. L., \& Jiménez-Sarmiento, A. S. (2011). Overview of musculoskeletal pain in the flamenco dancer. Rehabilitacion, 45(2), 117-121. https://doi.org/10.1016/j.rh.2011.03.003

Castillo-López, J. M., Munuera-Martínez, P. V., Algaba-Guisado, C., Reina-Bueno, M., Salti-Pozo, N., \& Vargas-Macías, A. (2016). Pathologic disorders of the foot in professional female flamenco dancers. Journal of the American Podiatric Medical Association, 106(1), 54-59.

Castillo-López, J. M., Munuera-Martínez, P. V., Gómez-Benítez, M. A., Pérez-García, L., Salti-Pozo, N., \& Palomo, I. C. (2017). El dolor patelofemoral en el baile flamenco y su relación con el pie. Revista del Centro de Investigación Flamenco Telethusa, 10(12), 62-67. https://doi. org/10.23754/telethusa.101204.2017

Castillo-López, J. M., Palomo, I. C., Munuera-Martínez, P. V, Ramos-Ortega, J., \& Domínguez-Maldonado, G. (2015). Análisis podológico del zapateado flamenco Podiatric analysis of flamenco dancing footwork, Revista del Centro de Investigación Flamenco Telethusa, 8(9), 11-18.

Castillo-López, J. M., Vargas-Macías, A., Domínguez-Maldonado, G., Lafuente-Sotillos, G., Ramos-Ortega, J., Palomo-Toucedo, I. C., ... \& Munuera-Martínez, P. V. (2014). Metatarsal pain and plantar hyperkeratosis in the forefeet of female professional flamenco dancers. $\mathrm{Me}$ dical Problems of Performing Artists, 29(4), 193-197.

Duthon, V. B., Charbonnier, C., Kolo, F. C., Magnenat-Thalmann, N., Becker, C. D., Bouvet, C., ... \& Menetrey, J. (2013). Correlation of clinical and magnetic resonance imaging findings in hips of elite female ballet dancers. Arthroscopy: The Journal of Arthroscopic \& Related Surgery, 29(3), 411-419.

Echegoyen, S., Aoyama, T., \& Rodríguez, C. (2013). Zapateado technique as an injury risk in Mexican folkloric and Spanish dance: an analysis of execution, ground reaction force, and muscle strength. Medical Problems of Performing Artists, 28, 80-83.

Echegoyen, S., Acuña, E., \& Rodríguez, C. (2010). Injuries in students of three different dance techniques. Medical Problems of Performing Artists, 25, 72-74

Encarnacion, M. L., Meyers, M. C., Ryan, N. D., \& Pease, D. G. (2000). Pain Coping Styles of Ballet Performers. Journal of Sport Behavior, 23(1), 20-32.

Eustergerling, M., \& Emery, C. (2015). Risk factors for injuries in competitive Irish dancers enrolled in dance schools in Calgary, Canada. $\mathrm{Me}$ dical Problems of Performing Artists, 30(1), 26-29.

Forczek, W., Baena-Chicón, I., \& Vargas-Macías, A. (2016). Variación de la posición del centro de gravedad en una bailaora profesional durante el zapateado flamenco. Revista del Centro Investigación Flamenco Telethusa, 9(10), 30-36.

Gómez, S., Santonja, F., Vargas, A., \& Canteras, M. (2010). Antecedentes de algias vertebrales en bailarinas. En II Congreso Internacional de Ciencias del Deporte de la UCAM. Área de actividad física y salud. CCD 5(13 Sup) (pp. 11-62).

Heras, B. (2010). La danza en las leyes educativas españolas contemporáneas. Cuestiones Pedagógicas. Revista de Ciencias de la Educación, 20 307-327.

Hoogenboom, B. J., \& Voight, M. L. (2017). Rolling revisado: utilización del rolling para valorar y tratar la coordinación y control neuromuscular del core y extremidades en atletas. Revista del Centro de Investigación Flamenco Telethusa, 10(12), 19-35. https://doi.org/10.23754/ telethusa.101201.2017

Koutedakis, Y., \& Jamurtas, A. (2004). The dancer as a performing athlete. En Sports Medicine (Vol. 34, pp. 651-661).

Mainwaring, L. M., \& Finney, C. (2017). Psychological risk factors and outcomes of dance injury: A systematic review. Journal of Dance Medicine \& Science, 21(3), 87-96.

Mainwaring, L. M., Hutchison, M., Bisschop, S. M., Comper, P., \& Richards, D. W. (2010). Emotional response to sport concussion compared to ACL injury. Brain injury, 24(4), 589-597

Mayers, L., Judelson, D., \& Bronner, S. (2003). The prevalence of injury among tap dancers. Journal of Dance Medicine \& Science, 7(4), 121-125.

McGuinness, D., \& Doody, C. (2006). The injuries of competitive Irish dancers. Journal of Dance Medicine \& Science, 10(1-2), 35-39.

Navarro, J. L., \& Pablo, E. (2005). El baile flamenco. Una aproximación histórica. Córdoba: Almuzara.

Nogareda, S., Salas, C., \& Parra, J. M. (2007). La salud laboral en el arte flamenco. Notas Técnicas de Prevención.

Omino, K., \& Hayashi, Y. (1992). Preparation of dynamic posture and occurrence of low back pain. Ergonomics, 35(5-6), 693-707.)

Paparizos, A., Tripp, D., Sullivan, M., \& Rubenstein, M. (2004). Catastrophizing and pain perception in recreational ballet dancers. Journal of Sport Behavior, 28(1), 35-50.

Pedersen, M. E., Wilmerding, M. V., Kuhn, B. T., \& Enciñias-Sandoval, E. (2001). Energy requirements of the american professional flamenco dancer. Medical Problems of Performing Artists, 16(2), 47-52.

Pedersen, M. E., \& Wilmerding, V. (1998). Injury profiles of student and professional flamenco dancers. Journal of Dance Medicine y Science, 2(3), 108-114.

Petrie, T. A. \& Falkstein, D. L. (1998) Methodological, measurement, and statistical issues in research on sport injury prediction. Journal of Applied Sport Psychology, 10(1), 26-45, DOI: 10.1080/1041320 9808406376

Prokop L. The significance of pain in sport. Kinesiology. 2000;32(1):77- 84 Quer, A., \& Pérez, E. (2004). El Pie en el Flamenco. El Peu, 24(1), 8-14.

Reid, D. C. (1988). Prevention of hip and knee injuries in ballet dancers. Sports Medicine, 6(5), 295-307.

Rivera, D., Alexander, J., Nehrenz, G., \& Fields, B. (2012). Dancers Perceptions of Pain. Journal of Music and Dance, 2(1), 9-12. https://doi org/10.5897/JMD11.006

Russell, J. A. (2013). Preventing dance injuries: current perspectives. Open Access Journal of Sports Medicine, 4, 199

Russell, J. A., Shave, R. M., Yoshioka, H., Kruse, D. W., Koutedakis, Y., \& Wyon, M. A. (2010). Magnetic resonance imaging of the ankle in female ballet dancers en pointe. Acta Radiologica, 51(6), 655-661. 
Snow, R. E., \& Williams, K. R. (1994). High heeled shoes: their effect on center of mass position, posture, three-dimensional kinematics, rearfoot motion, and ground reaction forces. Archives of Physical Medicine and Rehabilitation, 75(5), 568-576.

Shybut, T. B., Rose, D. J., \& Strongwater, A. M. (2008). Second metatarsal physeal arrest in an adolescent flamenco dancer: a case report. Foot y Ankle International, 29, 859-862. https://doi.org/10.3113/ FAI.2008.0859

Sobrino, F. J., \& Guillén, P. (2017). Overuse Injuries in Professional Ballet: Influence of Age and Years of Professional Practice. Orthopaedic Journal of Sports Medicine, 5(6), 6-11. https://doi.org/10.1177/ 2325967117712704

Swain, C. T., Bradshaw, E. J., Ekegren, C. L., \& Whyte, D. G. (2019). The epidemiology of low back pain and injury in dance: a systematic review. Journal of Orthopaedic \& Sports Physical Therapy, 49(4), 239-252

Taylor, J., \& Taylor, S. (1998). Pain education and management in the rehabilitation from sports injury. The Sport Psychologist, 12(1), 68-88.

Turner, R., O'Sullivan, E., \& Edelstein, J. (2012). Hip dysplasia and the performing arts: Is there a correlation? Current Reviews in Musculoskeletal Medicine, 5(1), 39-45. https://doi.org/10.1007/s12178-011-9104-9
Vargas, A. (2009). El baile flamenco: estudio descriptivo, biomecánico y condición. Cádiz: Revista del Centro de Investigación Flamenco Telethusa.

Vargas, A., González, J. L., Mora, J., \& Gómez, S. (2008). La necesidad de la preparación física en el baile flamenco. / The need of fitness in Flamenco Dance. Revista del Centro de Investigación Flamenco Telethusa, 1(1), 4-6.

Vargas-Macías, A., Castillo, J. M., \& Fernández, J. del R. (2012). Inestabilidad del pie y tobillo en el baile flamenco. Revista del Centro de Investigación Flamenco Telethusa, 5(5), 40-45

Voloshin, A. S., Bejjani, F. J., Halpern, M., \& Frankel, V. H. (1989). Dynamic loading on flamenco dancers: a biomechanical study. Human Movement Science, 8(5), 503-513.

Weber, A. E., Bedi, A., Tibor, L. M., Zaltz, I., \& Larson, C. M. (2015). The Hyperflexible Hip: Managing Hip Pain in the Dancer and Gymnast. Sports Health, 7(4), 346-358. https://doi.org/10.1177/ 1941738114532431

Yoo, W. G. (2013). Comparison of the cervical extension angle and the upper trapezius muscle activity between overhead work and belowknee work. Journal of Physical Therapy Science, 25(10), 1289-1290. 